\title{
THE INTEGRATED CAUCHY FUNCTIONAL EQUATION: SOME COMMENTS ON RECENT PAPERS
}

\author{
C. R. RAO,* Pennsylvania State University \\ T. SAPATINAS, ${ }^{* *}$ University of Sheffield \\ D. N. SHANBHAG, ${ }^{* * *}$ University of Sheffield
}

\begin{abstract}
We make some comments on recent papers involving the integrated Cauchy functional equation or specialized versions of it, and reveal in particular that these papers give an inaccurate picture of the current state of the literature on the topic.

LAU-RAO THEOREM; DENY'S THEOREM; EXPOTENTIAL AND GEOMETRIC DISTRIBUTIONS; POISSON PROCESS

AMS 1991 SUBJECT CLASSIFICATION: PRIMARY 62E10

Rao (1983), Rao and Shanbhag (1986) and several others have observed that various results in characterization theory and applied probability involving the integrated Cauchy functional equation or its variants follow as obvious corollaries of the Lau-Rao theorem or Deny's theorem or extended versions of these results given more recently by Davies and Shanbhag (1987). In many cases, the cited results on functional equations provide results that are much stronger than those appearing in the literature. It is unfortunate that, in spite of the exposure given to these results, one still comes across papers on the subject that make no reference to these or make misleading statements on what they imply. In this short communication we discuss some of the most recent contributions that fall into this category.
\end{abstract}

\section{Introduction}

\section{Chukova and Dimitrov (1992)}

Chukova and Dimitrov (1992) have shown that there exist random variables $X$ not exponentially or geometrically distributed, such that

$$
\boldsymbol{P}(X-b \geqq x \mid X \geqq b)=\boldsymbol{P}(X \geqq x)
$$

for all $x>0$ and infinitely many different values of $b$. They called the distributions of such random variables almost-lack-of-memory distributions (ALMD), since they almost have the lack of memory property. From their paper, it is evident that they are aware neither of Lau and Rao (1982) nor of Marsaglia and Tubilla (1975). The solution to the class of ALMD that these authors give can be seen to be a trivial corollary to the Lau-Rao theorem. Indeed the

\footnotetext{
Received 29 October 1993.

* Postal address: Department of Statistics, Pennsylvania State University, University Park, PA 16802, USA.

** Present address: Department of Mathematical Statistics and Operational Research, Laver Building, Exeter University, Exeter, EX44QE, UK.

*** Postal address: School of Mathematics and Statistics, The University, Sheffield S10 2TN, UK.

Research supported by US Army Research Grant DAA HO4-93-G-0030.
} 
result here is of an elementary nature and does not require anything as powerful as the Lau-Rao theorem to deal with it. However, references to the Lau-Rao theorem and its corollary due to Marsaglia and Tubilla (1975) would have exposed the link between these latter results and the cited result of Chukova and Dimitrov. We reproduce below the statement of the Lau-Rao theorem for ready reference. (For a probabilistic proof of the Lau-Rao theorem based on exchangeability, we refer the reader to Alzaid et al. (1987).)

The Lau-Rao theorem. Let $f$ be a non-negative real locally integrable Borel-measurable function on $\mathbb{R}_{+}$, other than a function which is identically zero a.e. $[L]$, such that it satisfies

$$
f(x)=\int_{\mathbb{R}_{+}} f(x+y) d \mu(y) \quad \text { for a.a. }[L] \quad x \in \mathbb{R}_{+}
$$

for some $\sigma$-finite measure $\mu$ with $\mu(\{0\})<1$ (yielding trivially that $\mu\left(\{0\}^{c}\right)>0$ ), where $L$ corresponds to Lebesgue measure. Then, either $\mu$ is arithmetic with some span $\lambda$ and

$$
f(x+n \lambda)=f(x) b^{n}, \quad n=0,1, \cdots \quad \text { for a.a. }[L] \quad x \in \mathbb{R}_{+}
$$

with $b$ such that

$$
\sum_{n=0}^{\infty} b^{n} \mu(\{n \lambda\})=1
$$

or $\mu$ is non-arithmetic and

with $\eta$ such that

$$
f(x) \propto \exp (-\eta x) \quad \text { for a.a. }[L] \quad x \in \mathbb{R}_{+}
$$

$$
\int_{\mathbb{R}_{+}} \exp (-\eta x) d \mu(x)=1 .
$$

(Here a.e. and a.a. stand for 'almost everywhere' and 'almost all' respectively.)

It may be worth pointing out at this stage that Dimitrov and Khalil (1990) also contains results of which stronger versions follow trivially from known results on functional equations. This has been pointed out explicitly or implicitly by Shanbhag (1991) and van Harn and Steutel (1991).

\section{Huang et al. (1993)}

Huang et al. (1993) have recently given, amongst other things, some variants and extended versions of the following theorem of Gupta and Gupta (1986).

Theorem. Let $\left\{S_{n}, n \geqq 1, F(x)\right\}$ be a renewal process where $F(x)$ is continuous. Let $G(x)$ be a monotone non-decreasing function having a non-lattice support on $x \geqq 0$ with $G(0)=0$ and $\int_{0}^{\infty} e^{-\xi x} d G(x)<\infty$ for all $\xi>0$. Suppose further that $g(t)=\boldsymbol{E}(G(u(t)))<\infty$ (where $u(t)$ is the forward recurrence time at $t$ ) for all $t$. If $g(t)$ is constant, then $\left\{S_{n}\right\}$ is a Poisson process.

Gupta and Gupta (1986) proved their theorem by using a result which is due to Shimizu (1978), (1979). However, Rao and Shanbhag (1989) have revealed that if the Lau-Rao theorem is used instead of Shimizu's result, then a more general version of the above theorem is obtained. In particular, as Rao and Shanbhag (1989) mentioned, if one replaces the assumption that $F$ is continuous by the weaker assumption that $F(0)=0$ subject to an alteration that $G$ is now assumed to be right continuous, and if one drops the condition that $\int_{0}^{\infty} e^{-\xi x} d G(x)<\infty$ for all $\xi>0$, then the theorem still holds. This follows as it is easily seen that the equation $g(t)=c$ (constant) is equivalent to

$$
c(1-F(x))=\int_{\mathbb{R}_{+}}(1-F(x+y)) d G(y), \quad x \in \mathbb{R}_{+},
$$


which implies that $F$ is exponential. Incidentally, the differentiability of $G$, implicitly assumed in the proof given by Gupta and Gupta (1986), is not necessary; this is an observation of Rao and Shanbhag (1989).

Huang et al. (1993), in establishing Theorems 3 and 4 of the paper, implicitly used the cited result of Shimizu and a corollary to it (i.e. Corollary 1 in Huang et al. (1993)). Obviously, if one uses the Lau-Rao theorem instead of the restrictive result of Shimizu and its corollary, more general versions of the Huang et al. (1993) results follow. Unfortunately, these latter authors do not make any reference to Lau and Rao (1982) and Rao and Shanbhag (1989), and so in presenting their results they once again make unnecessary assumptions essentially as in Gupta and Gupta (1986). Also, these authors could have benefited from the letter to the editor of Lau and Prakasa Rao (1992) and would have known, amongst other things, that a version of the Lau-Prakasa Rao result was given earlier in Kakosyan et al. (1984).

We should also mention here that both Gupta and Gupta (1986) and Huang et al. (1993) have missed the paper of Isham et al. (1975) in which a result relevant to the findings of these papers was arrived at.

\section{Witte (1993)}

This author mentions that Rao and Shanbhag (1986) have shown that the Lau-Rao theorem and various corollaries to it are implicitly contained in Deny's theorem. However, if one scrutinizes the paper of Rao and Shanbhag (1986), then it is not difficult to realize that what is claimed by Witte (1993) is untrue. Indeed, to get the Lau-Rao theorem from Deny's theorem, Rao and Shanbhag (1986) have used an inequality satisfied by the function relative to the functional equation. This inequality is a result of Davies and Shanbhag (1987) and it is a key to their investigations. The inequality in question is not a trivial result, although it has an elegant proof based on exchangeable random variables. Witte (1993) seems to be overlooking this point. We may, however, mention here that if, in the integrated Cauchy functional equation (1), the function $f$ is bounded and the measure $\mu$ is finite, then the Lau-Rao theorem can be easily obtained via Deny's theorem as revealed by Fosam et al. (1993). In view of this, the Marsaglia and Tubilla (1975) characterization of the exponential distribution follows as an easy consequence of Deny's theorem. Also, some of the recent results on integral equations relative to the real line or the set of integers follow trivially from Deny's theorem.

We are not claiming here that Deny's method cannot be adapted to deal with the Lau-Rao theorem or its corollaries. What we are stating is that these latter results are not obvious corollaries of the result which is referred to as Deny's theorem in the literature. To illustrate our point, we consider the following very simple example:

Example 1. Consider the system of equations

$$
v_{n}=\sum_{m=0}^{\infty} w_{m} v_{n+m}, \quad n=0,1, \cdots
$$

studied by Shanbhag (1977) with assumptions as stated by him. To get Shanbhag's result as a corollary to Deny's theorem, one should first demonstrate that $\left\{v_{n}: n=0,1, \cdots\right\}$ of (2) is extendable to a non-negative sequence $\left\{v_{n}^{*}: n=0, \pm 1, \pm 2, \cdots\right\}$ such that the following system of equations is valid:

$$
v_{n}^{*}=\sum_{m=0}^{\infty} w_{m} v_{n+m}^{*}, \quad n=0, \pm 1, \pm 2, \cdots
$$

The proof of the assertion does not seem to be trivial.

In the case of more general semigroups, the problem becomes still more complex and, as mentioned by Davies (1993), one requires more complicated arguments to get the results than those that are sufficient to obtain Deny's theorem. That, in general, the function in the 
integral equation corresponding to an embeddable semigroup cannot be extended to have an integral equation corresponding to a group is shown by the following example.

Example 2. Let $f$ be a density function on $\mathbb{R}_{+}^{2}$ satisfying

$$
f\left(x_{1}, x_{2}\right)=\lambda \int_{0}^{\infty} \int_{0}^{\infty} f\left(x_{1}+y_{1}, x_{2}+y_{2}\right) d y_{1} d y_{2} \quad\left(x_{1}, x_{2}\right) \in \mathbb{R}_{+}^{2},
$$

where $\lambda>0$. (In other words, $f$ is the density corresponding to a distribution that is absolutely continuous and is concentrated on $\mathbb{R}_{+}^{2}$, with failure rate $\lambda$.) The solution to (3) has been identified by Puri and Rubin (1974) (see also Davies and Shanbhag (1987)) and it is of the form

$$
f\left(x_{1}, x_{2}\right)=\lambda \boldsymbol{E}\left(e^{-x_{1} z_{1}-x_{2} z_{2}}\right) \quad\left(x_{1}, x_{2}\right) \in \mathbb{R}_{+}^{2},
$$

where $\left(Z_{1}, Z_{2}\right)$ is a vector of positive random variables such that $Z_{1} Z_{2}=\lambda$ a.s. If we take $Z_{1}$ here for example as any positive random variable for which the moment generating function $M(t)$ is not defined for some or all positive $t$ (e.g. a log-normal random variable, a positive stable random variable or a gamma random variable) and take $Z_{2}=\lambda / Z_{1}$, then, we see that $f$ in (4) is well-defined and is as required. However, in this case, we cannot extend the function $f$ to have a new function $f^{*}$ on $\mathbb{R}^{2}$ such that $f^{*}$ satisfies the integral equation on $\mathbb{R}^{2}$.

\section{Comments on some other papers}

There are several other papers which involve implicitly or explicitly the integrated Cauchy functional equation and the relevant results therein follow as corollaries to the Lau-Rao theorem. For example, Fosam and Shanbhag (1994) show that the strong memoryless property characterizations of the exponential and geometric distributions that are corollaries to the theorem in question yield stronger versions of a result of Liang and Balakrishnan (1992).

Recently, Pakes and Khattree (1992) considering a length-biased model obtained a characterization of the generalized gamma family. The functional equation (5.3) in Pakes and Khattree (1992) is, obviously, a special case of the equation in the Lau-Rao theorem and so the characterization in question immediately follows. However, Pakes and Khattree seem not to have noticed this.

In concluding, we may point out that many other results from the literature could now be improved upon using the cited results on functional equations. A forthcoming monograph of Rao and Shanbhag will address this issue; we refer also to Sapatinas (1993) for some specific applications of the Lau-Rao theorem, giving in particular characterizations of Pareto distributions.

\section{References}

Alzaid, A. A., Rao, C. R. And Shanbhag, D. N. (1987) Solution of the integrated Cauchy functional equation on the half line using exchangeability. Sankhya A 49, 189-194.

Chukova, S. AND Dimitrov, B. (1992) On distributions having the almost-lack-of-memory property. J. Appl. Prob. 29, 691-698.

Davies, P. L. (1993) Review of Ramachandran and Lau (1991). Math. Rev. (93c:60016), 1518-1519.

Davies, P. L. AND Shanbhag, D. N. (1987) A generalization of a theorem of Deny with applications in characterization theory. Quart. J. Math. Oxford (2) 38, 13-34.

Dimitrov, B. AND KhaliL, Z. (1990) On a new characterization of the exponential distribution related to a queueing system with an unreliable server. J. Appl. Prob. 27, 221-226.

Fosam, E. B., Rao, C. R. and Shanbhag, D. N. (1993) Comments on some results involving the integrated Cauchy functional equation. Statist. Prob. Lett. 17, 299-302. 
Fosam, E. B. AND Shanbhag, D. N. (1994) Certain characterizations of exponential and geometric distributions. J. R. Statist. Soc. B 56, 157-160.

Gupta, P. L. AND GuPta, R. C. (1986) A characterization of the Poisson process. J. Appl. Prob. 23, 233-235.

VAN HaRn, K. AND Steutel, F. W. (1991) On a characterization of the exponential distribution. $J$. Appl. Prob. 28, 947-949.

Huang, W. J., LI, S. H. AND Su, J. C. (1993) Some characterizations of the Poisson process and geometric renewal process. J. Appl. Prob. 30, 121-130.

Isham, V., Shanbhag, D. N. And Westcott, M. (1975) A characterization of the Poisson process using forward recurrence times. Math. Proc. Camb. Phil. Soc. 78, 513-515.

Kakosyan, A. V., Klebanov, L. B. And Melamed, J. A. (1984) Characterization of Distributions by Method of Intensively Monotonic Operators. Lecture Notes in Mathematics 1088, Springer-Verlag, New York.

LaU, K. S. And Prakasa RaO, B. L. S. (1990) Characterization of the exponential distribution by the relevation transform. J. Appl. Prob. 27, 726-729.

Lau, K. S. And Prakasa Rao, B. L. S. (1992) Letter to the editor. J. Appl. Prob. 29, 1003-1004.

LAU, K. S. AND RAO, C. R. (1982) Integrated Cauchy functional equation and characterizations of the exponential law. Sankhyā A 44, 72-90.

Liang, T. C. AND BALAKrishnan, N. (1992) A characterization of the exponential distribution through conditional independence. J. R. Statist. Soc. B 54, 269-271.

Marsaglia, G. and Tubilla, A. (1975) A note on the lack of memory property of the exponential distribution. Ann. Prob. 3, 352-354.

Pakes, A. G. AND Khattree, R. (1992) Length-biasing, characterizations of laws and the moment problem. Austral. J. Statist. 34, 307-322.

PURI, P. S. AND RUBIN, H. (1974) On a characterization of the family of distributions with constant multivariate failure rates. Ann. Prob. 2, 738-740.

RAO, C. R. (1983) An extension of Deny's theorem and its applications to characterization of probability distributions. In A Festchrift for Eric Lehmann, ed. P. J. Bickel et al., pp. 348-366, Wadsworth, Monterey, CA.

RaO, C. R. AND Shanbhag, D. N. (1986) Recent results on characterization of probability distributions: a unified approach through extensions of Deny's theorem. Adv. Appl. Prob. 18, 660-678.

RaO, C. R. AND Shanbhag, D. N. (1989) Recent advances on the integrated Cauchy functional equation and related results in applied probability. In Papers in Probability, Statistics and Mathematics in Honor of S. Karlin, ed. T. W. Anderson et al., pp. 239-253, Academic Press, New York.

Sapatinas, T. (1993) Characterization and Identifiability Results in some Stochastic Models. Unpublished Ph.D. Thesis, Sheffield University.

ShanbhaG, D. N. (1977) An extension of the Rao-Rubin characterization of the Poisson distribution. J. Appl. Prob. 14, 640-646.

Shanbhag, D. N. (1991) Review of Dimitrov and Khalil (1990) Math. Rev. (91j:60147), 5590.

SHIMIZU, R. (1978) Solution to a functional equation and its applications to some characterization problems. Sankhyā A 40, 319-332.

Shimizu, R. (1979) On a lack of memory property of the exponential distribution. Ann. Inst. Statist. Math. 31, 309-313.

WITTE, H.-J. (1993) Some characterizations of exponential or geometric distributions in a nonstationary record value model. J. Appl. Prob. 30, 373-381. 